\title{
Disintegration of Antimony-124
}

\author{
By Irving Feister and Leon F. Curtiss
}

\begin{abstract}
The radiations from radio antimony of 60-day half-period $\mathrm{Sb}^{124}$, have been investigated in a magnetic lens electron spectrometer. The beta-ray spectrum was found to have two components with maximum energies of $0.57 \pm 0.02$ and $2.24 \pm 0.05$ million electron volts. The gamma-ray spectrum has two lines, one at $0.60 \pm 0.01$ and the other at $1.69 \pm 0.02$. million electron volts. It appears quite certain that the $0.60 \mathrm{Mev}$ gamma ray originates from $\mathrm{Sb}^{124}$, and therefore these measurements substantiate the disintegration scheme proposed by Meyerhof and Scharff-Goldhaber [1], ${ }^{1}$ with a small revision of energies.
\end{abstract}

\section{Introduction}

The radiations from 60-day $\mathrm{Sb}^{124}$ have been studied by several investigators. Hales and Jordan [2] used a $180^{\circ}$ spectrometer to study the beta spectrum of $\mathrm{Sb}^{124}$. They report the presence of two components with maximum energies at $0.74 \pm 0.03$ and $2.45 \pm 0.07$ million electron volts (Mev). Miller and Curtiss [3], using a thin magnetic lens spectrometer, also observed a complex beta spectrum, but with maximum energies at 0.53 and $2.25 \mathrm{Mev}$.

The most recent investigation of the beta and gamma radiations is that of Meyerhof and ScharffGoldhaber [1] by means of coincidence-absorption measurements. They report the presence of two beta-ray components with energies of 0.7 and 2.4 Mev; and two gamma rays with energies of 0.6 and 1.7 Mev. A table summarizing the results of previous studies of $\mathrm{Sb}^{124}$ is also given by them.

Although the gamma radiations from $\mathrm{Sb}^{124}$ had been studied by a number of investigators prior to these authors, none of them had reported the presence of a $0.6-\mathrm{Mev}$ gamma ray. With one exception, these prior reports indicated only one gamma ray with energy about $1.7 \mathrm{Mev}$. The present paper provides further information regarding the origin of this $0.6-\mathrm{Mev}$ gamma ray.

The energies of the beta and gamma rays from $\mathrm{Sb}^{124}$ reported here were obtained by means of a spectrometer of the thin magnetic lens type. This instrument has been described by Miller and Curtiss [4]. The results obtained are in partia] agreement with those previously reported, but differ significantly in certain respects. It is believed that the type of spectrometer used by the authors provides a more accurate determination of the $\mathrm{Sb}^{124}$ beta and gamma energies than can be obtained from absorption measurements such as used by Meyerhof and Scharff-Goldhaber [1].

\section{Preparation of Sources}

To obtain radioactive $\mathrm{Sb}^{124}$ for these experiments, a sample of antimony, estimated to be about 99.95 percent pure, was prepared at the Bureau of Standards. This sample was then sealed in a quartz capsule and irradiated with slow neutrons in the Clinton Laboratories pile at Oak Ridge, Tenn. Since ordinary antimony consists of about 56 percent of $\mathrm{Sb}^{121}$ and 44 percent of $\mathrm{Sb}^{123}$, the resultant reactions $\operatorname{Sb}^{121}(n, \gamma)$ and $\mathrm{Sb}^{123}(n, \gamma)$ produce a mixture of 2.8-day $\mathrm{Sb}^{122}$ and 60-day $\mathrm{Sb}^{124}[5]$.

The preparation of the beta and gamma sources used in the spectrometer has been described in detail in a previous paper [6]. The same methods were used here. To prepare a beta source, the antimony crystals were first ground up with a Carboloy mortar and pestle to a fine powder. A small amount of this powder was then deposited on a thin aluminum foil moistened with dilute glyptal solution.

The gamma source was prepared by placing a relatively large amount of the antimony crystals

\footnotetext{
${ }^{1}$ Figures in brackets indicate the literature references at the end of this paper.
} 
in a brass capsule, the opening of which was then sealed with Apiezon-Q wax. A radiator of uranium foil $\left(42 \mathrm{mg} / \mathrm{cm}^{2}\right)$ was used to convert the gamma rays from $\mathrm{Sb}^{124}$ into photoelectrons whose energy can be measured in the spectrometer.

\section{Beta-Ray Spectrum of $\mathrm{Sb}^{124}$}

The beta-ray spectrum was determined about 6 months after receipt of the source from Oak Ridge. The amount of 2.8-day $\mathrm{Sb}^{122}$ left after this long an interval is completely negligible, so that the remaining activity must be due to 60-day $\mathrm{Sb}^{124}$. Figure 1 shows the beta spectrum ob-

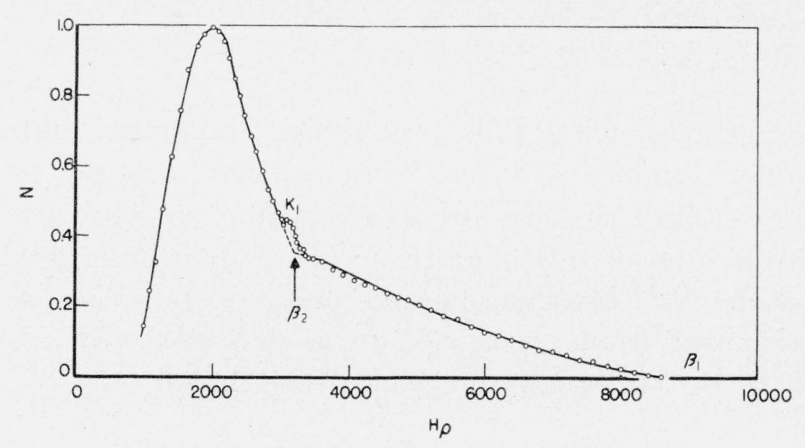

FIGURe 1. Primary beta-ray spectrum of $\mathrm{Sb}^{124}$ showing the intensity plotted against $H \rho$.

The $K_{1}$ peak is probably due to internal conversion electrons from a 0.60 Mev gamma ray.

tained. The quantity $N$ represents the relative intensity over equal intervals of $H \rho$ (gauss-cm). It is equal to the number of counts per minute in the Geiger-Müller counter divided by $H_{\rho}$.

The small $K_{1}$ peak in figure 1 is no doubt due to internal conversion electrons produced by a 0.60 Mev gamma ray, which will be discussed later. Omitting this peak, it is apparent that the beta spectrum of $\mathrm{Sb}^{124}$ consists of two components. The first part of the curve, up to the $K_{1}$ peak, is due to the sum of the two components; the remainder of the curve, beyond the $K_{1}$ peak, is due to the higher-energy component alone. Unfortunately, the $K_{1}$ peak occurs in the vicinity of the "break" in the beta distribution curve, which indicates the end point of the lower-energy betaray group. This tends to obscure the location of this end point.

If we neglect the $K_{1}$ peak, however, and extrapolate the parts of the curve immediately preceding and following this peak, their intersection is found to lie at $H_{\rho}=3,175 \pm 75$ gauss-cm. This corresponds to an energy of $0.57 \pm 0.02 \mathrm{Mev}$. The higher-energy component approaches the $H_{\rho}$ axis asymptotically, which makes its end point difficult to determine by direct inspection of figure 1. For this purpose, therefore, we make use of the Fermi plot shown in figure 2, which will be discussed in detail in section IV.

The Fermi plot again indicates clearly the presence of two beta-ray components. The $K_{1}$ peak of figure 1 has been omitted from figure 2 , as indicated by the central broken portion of the curve. The extrapolated portions of this Fermi plot give the end points of the two beta components as $0.58 \pm 0.02 \mathrm{Mev}$ (in agreement with the value obtained from fig. 1) and $2.24 \pm 0.05 \mathrm{Mev}$. These values are lower than those obtained by Hales and Jordan (0.74 and $2.45 \mathrm{Mev}$ ) [2], but agree within the limits of experimental error with those of Miller and Curtiss (0.53 and $2.25 \mathrm{Mev}$ ) [3].

The Fermi plot of figure 2 does not result in straight line curves, in contrast with the results of Hales and Jordan [2]. This, however, is not necessarily significant because of the following considerations:

1. The relatively long half-life of $\mathrm{Sb}^{124}$, together with the high maximum energy of its beta spectrum, indicates that the transitions involved are

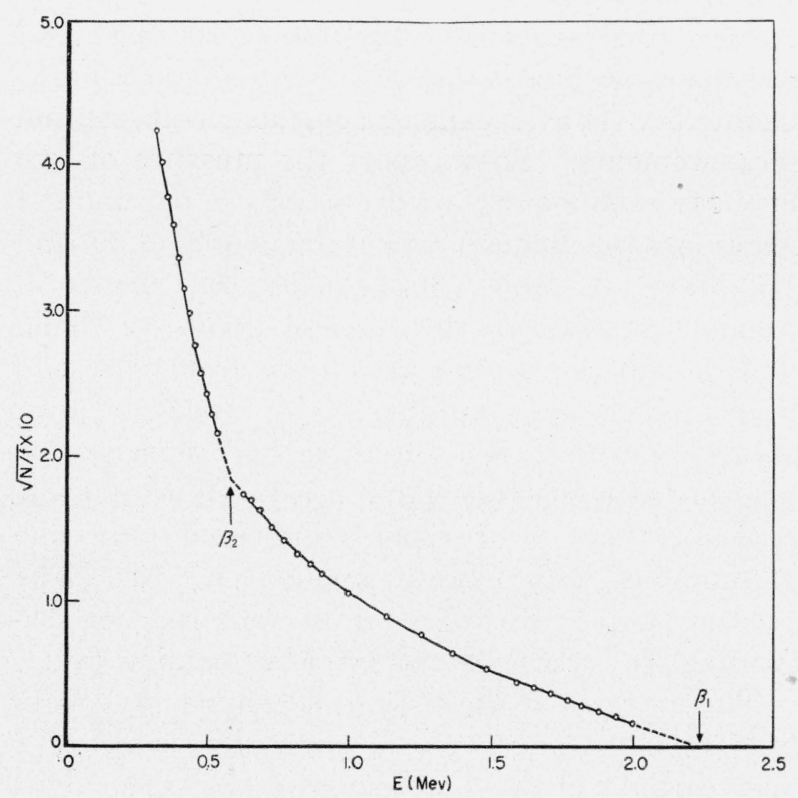

Figure 2. Fermi plot of the data shown in figure 1.

The original Fermi function $f(Z, \eta)$ was evaluated for $Z=51$, not the usual Kurie approximation. 
highly forbidden. The simple Fermi theory does not strictly apply to transitions of this type.

2. At the time the beta spectrum was measured the source had decayed through three half-lives, and its specific activity had decreased to about one-eighth of its initial value. Consequently, it was necessary to prepare a beta source whose thickness was such that the amount of absorption and scattering within the source cannot be considered entirely negligible. This would tend to distort the observed beta-ray spectrum.

\section{Fermi Plot}

The plot in figure 2 is based on Fermi's theory of beta-ray emission. Starting with eq 42 of Fermi's paper [7], let

$$
\begin{gathered}
K=g^{2} \frac{256 \pi^{4}}{[\Gamma(3+2 S)]^{2}} \frac{m^{5} c^{4}}{h^{7}}\left(\frac{4 \pi m c \rho}{h}\right)^{2 S} \\
Q^{*}{ }_{m n}=\int v_{m}{ }^{*} u_{n} d \tau .
\end{gathered}
$$

(See Fermi's paper for meaning of these expressions). For a given isotope, $K$ is constant.

Also, we have

where

$$
\sqrt{1+\eta_{0}^{2}}=E_{0}+1
$$

$\eta_{0}=$ maximum momentum of beta rays emitted in units of $m_{0} c$

$E_{0}=$ maximum energy of beta rays in units of $m_{0} c^{2}$

$m_{0}=$ rest mass of electron, and similarly,

$$
\sqrt{1+\eta^{2}}=E+1
$$

where $\eta$ and $E$ now refer to any electron with momentum $\eta$ (equal to $H \rho / 1700$ ) and energy $E$.

Substituting eq 1, 2, 3, and 4 in Fermi's eq 42 , the probability of emission of an electron with momentum between $\eta$ and $\eta+d \eta$ can be written

$$
P(\eta) d \eta=K\left|Q^{*}\right|^{2}\left[\eta^{2} F(Z, \eta)\right]\left(E_{0}-E\right)^{2} d \eta,
$$

where

$$
\begin{gathered}
F(Z, \eta)=\eta^{2 S} e^{\pi y}|\Gamma(1+S+i y)|^{2} \\
S=\sqrt{1-\left(\frac{Z}{137}\right)^{2}}-1 \\
y=\frac{Z}{137} \frac{\sqrt{1+\eta^{2}}}{\eta},
\end{gathered}
$$

and $Z$ is the atomic number.
According to Fermi, in case of "allowed" transitions the matrix element $Q^{*}$ in eq 5 is of the order of unity. For "forbidden" transitions, however, $\left|Q^{*}\right|^{2}<<1$. In thelatter case $Q^{*}$ probably depends on the momentum $\eta$ of the emitted beta rays, although the exact nature of this dependence is not known. As a first approximation, we shall assume that $\left|Q^{*}\right|^{2}$ is constant for all values of $\eta$.

Since the quantity $N$ in the beta spectrum of figure 1 is proportional to $P(\eta)$, we have from eq 5

where

$$
N \Delta \eta \propto f(Z, \eta)\left(E_{0}-E\right)^{2} \Delta \eta,
$$

$$
f(Z, \eta)=\eta^{2} F(Z, \eta) .
$$

Taking all the $\Delta \eta$ 's equal throughout the beta spectrum, eq 9 may be written

$$
\sqrt{\frac{\bar{N}}{f}} \propto\left(E_{0}-E\right)
$$

so that if we plot $\sqrt{N / f}$ against $E$, a straight-line curve is obtained which intersects the $E$ axis at $E=E_{0}$. This graphical representation of the beta-ray spectrum is commonly referred to as a Fermi plot or Kurie plot, and is frequently used to determine the end point of a beta distribution.

It should be remembered, however, that eq 11 is based on the assumption that $Q^{*}$ is independent of $E$, i. e.,

$$
\left|Q^{*}\right|^{2}=\text { constant }
$$

For allowed transitions, eq 12 is true at least approximately. For forbidden transitions, however, this is not necessarily true, and the Fermi plot need not be a straight line. Figure 2 indicates, therefore, that the beta transitions involved in the decay of $S b^{124}$ are of the forbidden typa.

The Fermi function $f(Z, \eta)$ used in making a Fermi plot is given by eq 10 and 6 . The factor $F(Z, \eta)$ in eq 5 is due to the effect of nuclear attraction upon the emitted beta ray. When $\eta>>/ 137$, $F(Z, \eta) \longrightarrow 1$. That is, for relatively high beta energies and light atoms, the effect of nuclear attraction is small.

A nonrelativistic treatment of the theory of beta emission leads to the expression

$$
F(Z, \eta)=\frac{2 \pi y}{1-e^{-2 \pi y}},
$$

whereas the more rigorous relativistic theory leads to eq 6. According to Kurie, et al. [8], eq 13 is adequate for all light elements up to about 
copper, $Z=29$ (provided $\eta$ is not $>>1$ ). Hence in this case the Fermi function can be written

$$
\left.\begin{array}{rl}
f(Z, \eta) & =\eta^{2} \frac{2 \pi y}{1-e^{-2 \pi y}} \\
y & =\frac{Z}{137} \frac{\sqrt{1+\eta^{2}}}{\eta}
\end{array}\right\}
$$

For $S b^{124}$, however, $Z=51$ and $\eta_{0} \cong 5$, so that the relativistic expression (eq 6 ) should be used. The Fermi function is then

$$
f(Z, \eta)=\eta^{2+2 S} e^{\pi y}|\Gamma(1+S+i y)|^{2} .
$$

As $Z=51$, we have from eq 7

$$
\left.\begin{array}{rl}
\frac{Z}{137} & =0.37 \\
S & =-0.072 \\
2+2 S & =1.86 \\
1+S & =0.93
\end{array}\right\}
$$

The numerical values of $Z / 137$ and $S$ in eq 16 have been rounded off to two significant figures. Substituting from eq 16 in eq 15 , we have for $\mathrm{Sb}^{124}$

$$
\left.\begin{array}{c}
f(\eta)=\eta^{1.86} e^{\pi y}|\Gamma(0.93+i y)|^{2} \\
y=0.37 \frac{\sqrt{1+\eta^{2}}}{\eta} .
\end{array}\right\}
$$

This is the function that was used in the Fermi plot of figure 2. The values of $f(Z, \eta)$ obtained from eq 17 have been compared with the corresponding values given by the nonrelativistic expression (eq 14). It is worth noting that the differences between the two values vary from 0 to 15 percent for the range of momenta considered here.

\section{Gamma-Ray Spectrum of $\mathrm{Sb}^{124}$}

The gamma-ray spectrum is shown in figure 3 . The distribution represented by the full curve was obtained about 1 month after receipt of the source from Oak Ridge. The activity of the 2.8-day $\mathrm{Sb}^{122}$ had by this time decayed to less than $10^{-3}$ of its initial amount, and was therefore negligible in comparison with the 60-day $\mathrm{Sb}^{124}$ activity. The continuous distributions in figure 3 are due to Compton recoil electrons, mostly from the

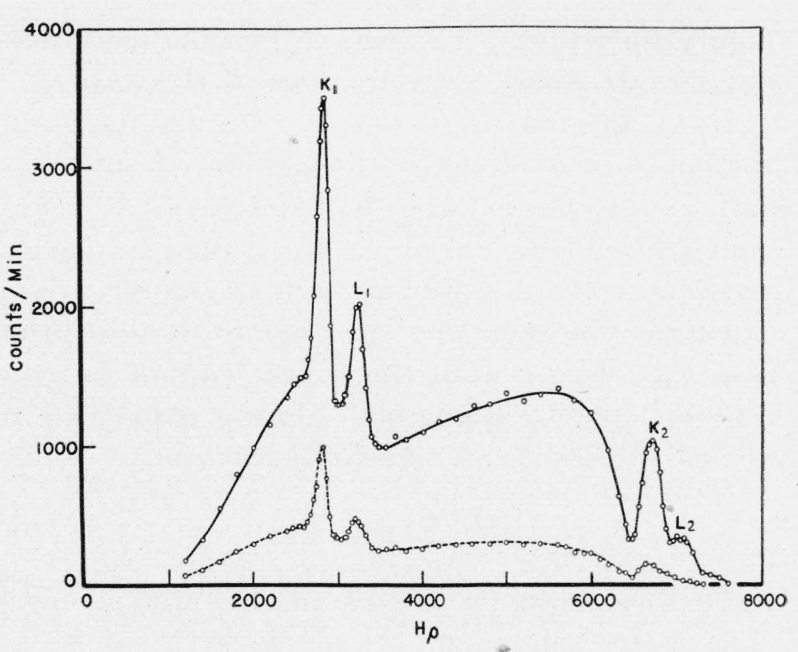

FIgURE 3. Secondary electron spectrum produced by $\mathrm{Sb}^{124}$ gamma rays in uranium.

$K_{1}, L_{1}$ are photoconversion lines of a 0.60 -Mev gamma ray; $K_{2}, L_{2}$ are due to a $1.69-\mathrm{Mev}$ gamma ray. Solid curve is gamma spectrum determined 1 month after preparation of source; broken curve is spectrum obtained 5 months after preparation of source.

brass capsule containing the source; whereas the superimposed peaks are due to photoelectrons from the uranium radiator (the photoelectrons from the brass capsule are relatively negligible in number) [6].

Figure 3 clearly shows the presence of two gamma rays, with energies of $0.60 \pm 0.01 \mathrm{Mev}$ and $1.69 \pm 0.02 \mathrm{Mev}$. These values are obtained by adding the $K$-binding energy of uranium $(0.115$ Mev) to the energies of the $K$-photoelectrons ejected from the uranium radiator by the two gamma rays, as indicated by the location of the $K_{1}$ and $K_{2}$ peaks. A further check may be obtained by adding the $L$-binding energy of uranium $(0.022 \mathrm{Mev})$ to the $L$-photoelectron energies indicated by the $L_{1}$ and $L_{2}$ peaks.

The energy of the $1.69 \mathrm{Mev}$ gamma ray agrees within the limits of experimental error with the value $1.70 \pm 0.02 \mathrm{Mev}$ previously reported by Kruger and Ogle [9], and with the value $1.72 \pm 0.03$ Mev reported by Rall and Wilkinson [10]. The $0.60 \mathrm{Mev}$ gamma ray, however, has not been reported by any investigators prior to Meyerhof and Scharff-Goldhaber [1]. This is difficult to understand, since figure 3 indicates that it is even more intense than the $1.69 \mathrm{Mev}$ gamma ray.

Rall and Wilkinson [10] also reported a 0.61 Mev gamma ray, which they tentatively assigned to 30 -day $\mathrm{Te}^{122,124}$. Both half-life and identity of these isotopes were indicated as being in doubt. 
This suggested the possibility that the $0.60 \mathrm{Mev}$ gamma ray observed by us might be identical with the $0.61 \mathrm{Mev}$ gamma ray reported by Rall and Wilkinson. To check this possibility, a chemical separation was made of the antimony and tellurium in the source about 9 months after its receipt from Oak Ridge. The separated tellurium fraction was then found to emit no detectable radiation. Had there been a 30 -day $\mathrm{Te}^{122,124}$ gamma activity in the tellurium fraction, it would almost certainly have been observed. We may safely conclude, therefore, that the $0.60 \mathrm{Mev}$ gamma ray shown in figure 3 is not due to such an activity.

To determine whether the relative intensity of the 1.69 and $0.60 \mathrm{Mev}$ gamma rays remains constant with time, the gamma spectrum was redetermined 4 months (2 half-lives of $\mathrm{Sb}^{124}$ ) after its first measurement. This second determination is shown by the broken curve in figure 3. Comparison of the two curves indicates that the two gamma rays decay approximately at the same rate and are therefore probably both due to 60-day $\mathrm{Sb}^{124}$.

\section{Disintegration Scheme of $\mathrm{Sb}^{124}$}

Meyerhof and Scharff-Goldhaber [1] have recently determined the relative intensities of the beta and gamma rays of $\mathrm{Sb}^{124}$ by means of Geigercounter coincidences. Their measurements indicate that the relative intensity of the two betaray components is approximately $1: 1$, and that of the two gamma rays is about $2: 1$. According to them, the hard beta ray is followed by one gamma ray, whereas the soft beta ray is followed by two gamma rays. We were unable to make any accurate determination of relative beta- and gamma-ray intensities from our measurements. In general, however, the relative intensities indicated in figures 1 and 3 tend to agree with the values reported by these authors.

The beta- and gamma-ray energies determined by us, combined with the above data of Meyerhof and Scharff-Goldhaber, can be fitted into the disintegration scheme shown in figure 4. This is identical with the decay scheme proposed by them, except for the more accurate determination of the energies involved.

The authors thank E. W. Cannon and the Computation Laboratory of the National Bureau of

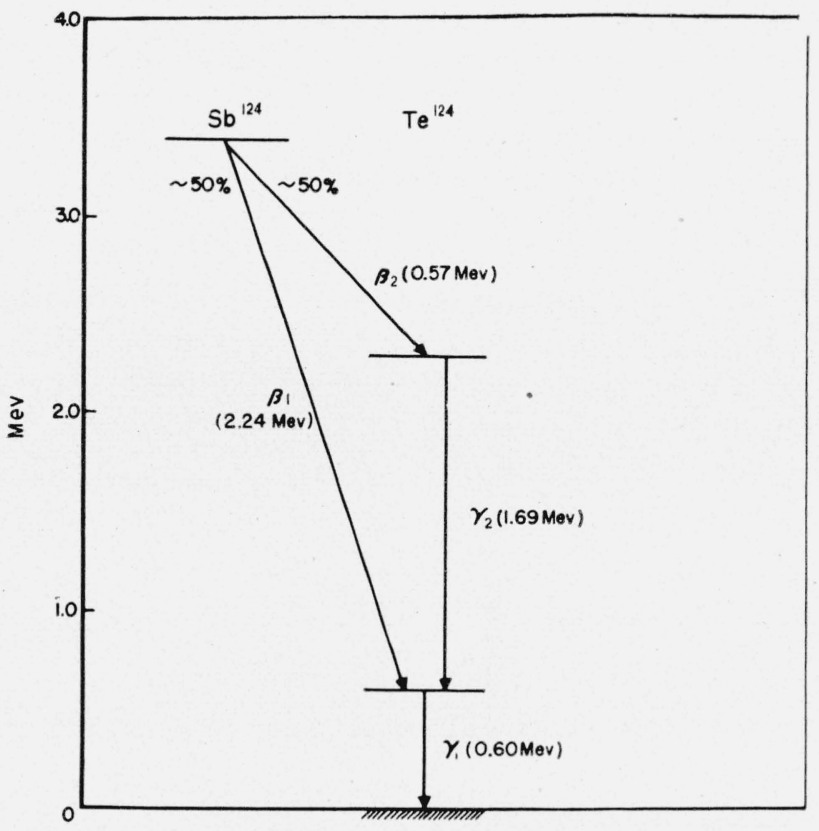

Figure 4. Suggested disintegration scheme for $\mathrm{Sb}^{124}$ based on the observed beta- and gamma-ray spectra and on the coincidence measurements reported by Meyerhof and Scharff-Goldhaber [1].

Beta transitions indicated above include energy equivalent of the electron mass.

Standards for the numerical evaluation of the original Fermi function for $Z-51$; and also $\mathrm{A}$. Schwebel for the chemical separation of antimony and tellurium in the source.

\section{References}

[1] W. E. Meyerhof and G. Scharfl-Goldhaber, Phys. Rev. 72, 273 (1947).

[2] E. B. Hales and E. B. Jordan, Phys. Rev. 64, 202 (1943).

[3] L. C. Miller and L. F. Curtiss, Phys. Rev. 70, 983 (1946).

[4] L. C. Miller and L. F. Curtiss, J. Research NBS 38, 359 (1947) RP1778.

[5] J. J. Livingood and G. T. Seaborg, Phys. Rev. 55, 414 (1939).

[6] I. Feister and L. F. Curtiss, J. Research NBS 38, 411 (1947) RP1781.

[7] E. Fermi, Z. Physik 88, 161 (1934).

[8] F. N. D. Kurie, J. R. Richardson, and H. C. Paxton, Phys. Rev. 49, 368 (1936).

[9] P. G. Kruger and W. E. Ogle, Phys. Rev. 67, 273 (1945).

[10] W. Rall and R. G. Wilkinson, Phys. Rev. \%1, 321 (1947).

Washington, October 22, 1947. 